\title{
Caspases como Alvo Terapêutico para o Tratamento DE Doenças NeUROdegenerativas
}

MARIA M. M. SANTOS ${ }^{*}$

\begin{abstract}
O controlo do número de células e tamanho dos tecidos, assim como a protecção dos organismos de células malignas, ocorre através de uma forma de morte celular programada, chamada apoptose. Quando ocorre uma desregulação da apoptose, podem surgir diversas doenças, onde se incluem as doenças neurodegenerativas (ex: doença de Huntington, doença de Alzheimer e doença de Parkinson). Dos diversos intervenientes que participam no processo que conduz à apoptose, destaca-se o papel fundamental de uma família de proteases de cisteína chamadas caspases.

Este artigo pretende fornecer aos leitores uma breve introdução sobre a apoptose e apresentar a contribuição da química terapêutica para o desenvolvimento de inibidores de caspases. Especial destaque será dado aos inibidores derivados de aceitadores de Michael, os quais ganharam grande relevância nos últimos anos.
\end{abstract}

A divisão celular é um evento fundamental durante o desenvolvimento de organismos multicelulares, sendo todos os dias substituídas milhões de células por segundo no corpo humano. O controlo do número de células e do tamanho dos tecidos, assim como a protecção dos organismos de células malignas ocorre através de uma forma de morte celular programada, chamada apoptose [1]. A desregulação da apoptose está associada a várias patologias incluindo o cancro, o enfarte de miocárdio e doenças neurodegenerativas (ex: doença de Alzheimer, doença de Huntington, doença de Parkinson e esclerose múltipla). Células que deveriam ser eliminadas podem tornar-se imortais nos casos em que existe uma falha na apoptose, podendo dar origem a doenças cancerígenas [2]. Pelo contrário, quando a apoptose ocorre de uma forma excessiva, demasiadas células são eliminadas conduzindo a danos graves em tecidos. Este é o caso do enfarte de miocárdio e das doenças neurodegenerativas [3]. Por estes motivos, estão a ser testadas várias aproximações terapêuticas para tratamento de doenças através da regulação da apoptose, nomeadamente através do

Research Institute for Medicines and Pharmaceutical Sciences (iMed.UL), Faculdade de Farmácia, Universidade de Lisboa Av. Prof. Gama Pinto, 1649-003 Lisboa, Portuga
E-mail: mariasantos@ff.ul.pt uso de fármacos que atacam alvos específicos na cascata de eventos que conduz à apoptose.

\section{Apoptose}

A apoptose foi descrita pela primeira vez em 1972 [4] e, desde essa data o número de publicações associadas à apoptose tem aumentado exponencialmente. No entanto, foi apenas nos anos 90 que começaram a surgir publicações relacionadas com moduladores da apoptose, tais como os receptores de superfície celular, as caspases, os factores mitocondriais ou a proteína p53.

A apoptose consiste numa cascata de eventos que conduz à morte celular $\mathrm{e}$ pode ocorrer por duas vias (Figura 1): a via extrínseca, mediada por receptores de morte, e a via intrínseca ou mitocondrial. Ambas as vias são controladas por uma família de proteases de cisteína, central para o processo

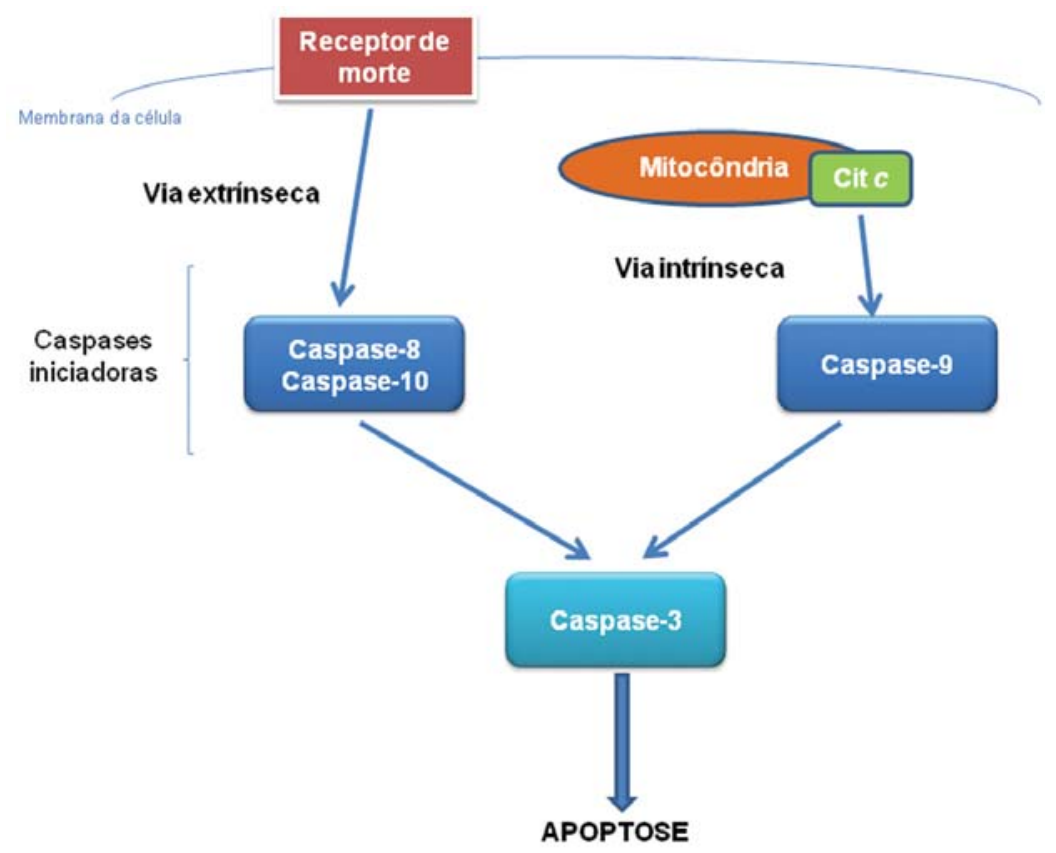

Figura 1 - Representação esquemática das duas vias principais da apoptose 
apoptótico, chamadas caspases [5-7]. A via extrínseca é controlada pela caspase-8 ou pela caspase-10 (caspases iniciadoras) e a via intrínseca ocorre através de libertação de citocromo $c$ da mitocôndria e consequente activação da caspase-9 (caspase iniciadora). Ambas as vias convergem na activação da caspase-3 (caspase executora), que conduz à morte celular [1].

\section{O Que São Então as Caspases?}

As caspases pertencem a um grupo de enzimas chamadas proteases de cisteína. Actualmente, são conhecidos 14 membros da família das caspases, dos quais 12 existem nos humanos [5-7]. A função de cada uma das caspases tem sido clarificada nos últimos anos, e é actualmente reconhecido que estas constituem novos alvos terapêuticos para doenças do sistema nervoso central, em que a morte celular ocorre através de um mecanismo de apoptose [8-9]. Consoante a sua função na cascata apoptótica, as caspases são divididas em caspases iniciadoras (caspases 2, 8, 9 e 10) ou caspases executoras (caspases 3,6 e 7), sendo estas últimas activadas pelas caspases iniciadoras durante a apoptose. Existem ainda as caspases $1,4,5,11$ e 12 que estão envolvidas na activação das citocinas.

Estão identificados vários reguladores de caspase naturais. Mas, nos últimos 20 anos, observou-se também um aumento exponencial de publicações relativas ao desenvolvimento de inibidores de caspases, tendo várias empresas farmacêuticas desenvolvido programas de investigação direccionados para a descoberta de fármacos que actuem sobre estes alvos terapêuticos [10-11].

\section{INIBIDORES DESENVOLVIDOS PARA AS Caspases}

As caspases representam uma das famílias de proteases mais específica, uma vez que requerem um resíduo de ácido aspártico na posição $\mathrm{P} 1$ do seu substrato, e requerem pelo menos três aminoácidos adicionais localizados nas posições P2-P4. Por esse motivo, um inibidor típico de caspase pode ser dividido em três componentes estruturais: o grupo químico que irá rea- gir com a cisteína do centro activo da enzima, o ácido aspártico na posição P1 e o componente P2-P4 peptidomimético (Esquema 1) [12].

Diferentes caspases possuem diferentes preferências para as posições P2 a P4. Por exemplo, as sequências tetrapeptídicas Asp-Glu-Val-Asp e Leu-Glu-Thr-Asp são sequências preferenciais para a caspase-3 e caspase-8, respectivamente [5]. No entanto, uma sequência dipeptídica truncada P2-P1 (por ex., Ala-Asp ou Val-Asp) é suficiente para obter inibidores selectivos e potentes para as caspases. A posição P1' do inibidor não contribui para a discriminação entre os membros da família das caspases, mas poderá determinar se o inibidor se liga reversivelmente ou irreversivelmente à posição S1' da enzima [11].

A maioria das estratégias utilizadas no desenho de inibidores de caspases envolveu modificação dos grupos

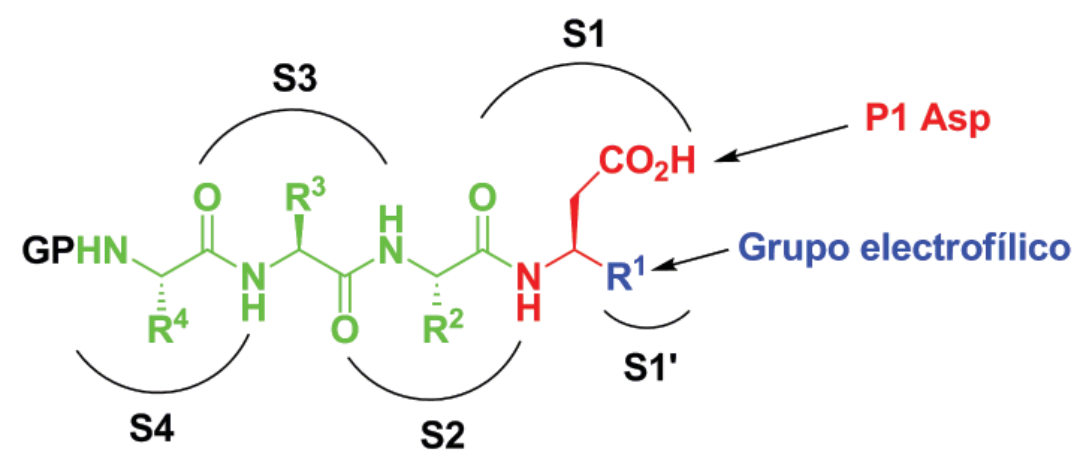

\section{GP = grupo protector}

Esquema 1 - Esquema de um inibidor de caspase típico. P1, P2, P3, P4 e P1' são as posições do inibidor que irão ocupar as posições S1, S2, S3, S4 e S1', respectivamente, do centro activo da enzima
HO.

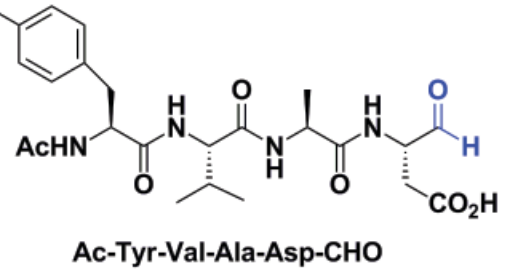<smiles>[R]NC(=O)C(=O)NC(C)C(=O)N[C@@H](CC(=O)O)C(=O)COc1c(F)c(F)cc(F)c1F</smiles>

Derivados de oxamilo
Ac-Tyr-Val-Ala-Asp-CHO electrofílicos de inibidores tetrapeptídicos conhecidos. Os primeiros inibidores de caspases desenvolvidos foram desenhados para actuar sobre a caspase-1. Só mais tarde começaram a ser desenhados inibidores para as caspases executoras -3 e -7 , bem como inibidores que actuassem sobre todas as caspases.

Por exemplo, no caso das caspases -1 e -3, vários inibidores foram baseados nos inibidores Ac-Tyr-Val-AlaAsp-CHO e Ac-Asp-Glu-Val-Asp-CHO (Figuras 2 e 3), respectivamente. Nomeadamente, aldeídos, nitrilos e cetonas peptídicas foram descritos como inibidores reversíveis, enquanto que derivados de cloro-, fluoro-, aciloxie diazometilcetonas foram descritos como inibidores irreversíveis [13-14]. Em particular, foram desenvolvidos potentes inibidores dipeptídicos irreversíveis, baseados em derivados de oxamilo [15] e em derivados de fluorometilcetonas (Figura 2) [16].

\footnotetext{
.
}

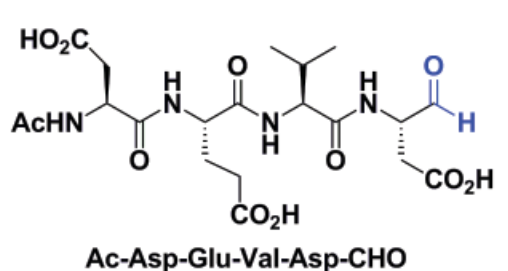<smiles>CC(C)C(NC(=O)OCc1ccccc1)C(=O)NC(CC(=O)O)C(=O)F</smiles>

MX1013 (Cbz-Val-Asp-CH ${ }_{2} \mathrm{~F}$ ) 
Devido aos problemas associados aos inibidores peptídicos, foram também desenvolvidos vários inibidores de caspases não peptídicos e peptidomiméticos (Figura 4). Em particular, entre os inibidores não peptídicos, destacam-se os derivados de isatina que não possuem um resíduo de ácido aspártico na posição P1 [18]. Já entre os inibidores peptidomiméticos, destacam-se os epóxidos azapeptídicos [19], assim como inibidores modificados nas posições P2 e P3 utilizando piridonas, pirimidonas e piridazinodiazepinas [13-14].

\section{Inibidores de Caspases Baseados em Aceitadores de Michael}

Nos últimos anos, os compostos derivados de aceitadores de Michael têm sido alvo de intensa investigação para o desenvolvimento de inibidores de proteases de cisteína. Destes, destacam-se os inibidores K-777 (CRA-3316) e o Ruprintivir (AG7088), desenvolvidos para tratar a doença de Chagas e como agente antirinoviral, respectivamente (Figura 5) [20].

No entanto, existem muito poucos exemplos descritos de inibidores de caspases derivados de aceitadores de Michael. Dos mais activos, destacamse os inibidores de caspases baseados em aza-péptidos [21] e sulfonamidas de isatina (Figura 6) [22].

Muito recentemente, o grupo de química terapêutica do iMed.UL (Research Institute for Medicines and Pharmaceutical Sciences, Faculdade de Farmácia de Lisboa) desenvolveu o primeiro estudo de estrutura-actividade de vinil sulfonas como inibidores de caspase-3. O estudo envolveu a síntese de uma série de vinil sulfonas contendo na cadeia de reconhecimento um aminoácido ou uma sequência dipeptídica (Figura 7). Todas as vinil sulfonas sintetizadas incluíram o ácido aspártico (Asp) na posição $\mathrm{P} 1$. A escolha do segundo aminoácido a introduzir na cadeia de reconhecimento foi feita com base nas sequências conhecidas como ideais para a caspase-3. Para o efeito, elegeram-se os aminoácidos valina (Val) e alanina (Ala) para a posição P2 dos novos inibidores. Finalmente, utilizou-se o grupo Z (benziloxicarbonilo ou $\mathrm{Cbz}$ ) ou o

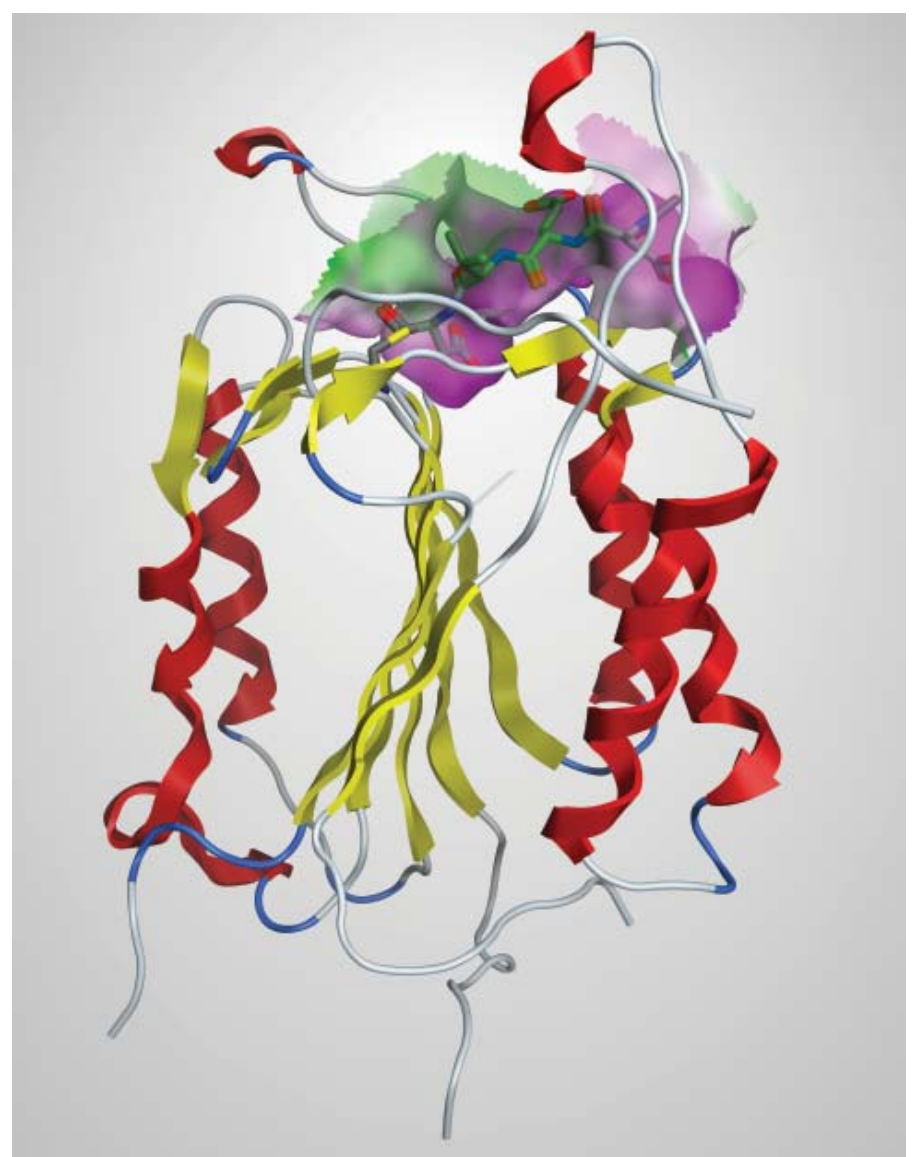

Figura 3 - Estrutura tridimensional do complexo da caspase-3 com o inibidor tetrapeptídico Ac-AspGlu-Val-Asp-CHO. Superfície do centro activo: rosa - zonas hidrofílicas; verde - zonas hidrofóbicas; branco - zonas neutras [17]<smiles>[R]N1C(=O)C(=O)c2cc(S(=O)(=O)N3CCC[C@H]3COc3ccccc3)ccc21</smiles><smiles>[R2]N[C@@H]([R1])C(=O)NN(CC(=O)O)C(=O)C1OC1[Y]</smiles>

Figura 4 - Exemplos de inibidores de caspases não peptídicos e peptidomiméticos

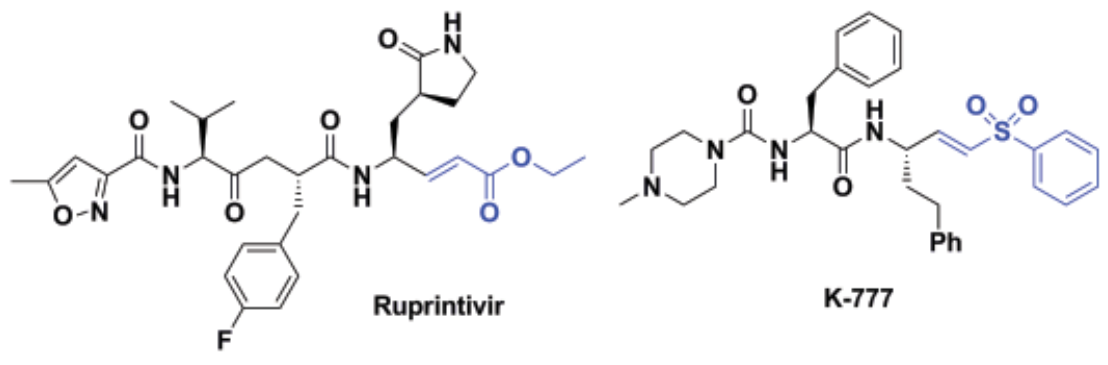

Figura 5 - Estruturas dos inibidores Ruprintivir e K-777<smiles>[R]NC([R])C(=O)NN(CC(=O)O)C(=O)/C=C/C([R])=O</smiles><smiles>[R1]N1C(=O)C(=C(C#N)C#N)c2cc(S([R1])(=O)=O)ccc21</smiles>

Figura 6 - Inibidores de caspases baseados em aceitadores de Michael 
grupo Fmoc (9-fluorenilmetoxicarbonilo) como grupos protectores (GP na Figura 7) do grupo amino $N$-terminal, e os grupos metilo ou fenilo na posição $P 1 '$ ' $\left(R^{1}\right.$ na Figura 7$)$. Os compostos foram avaliados in vitro para a caspase-3, tendo-se obtido valores de $\mathrm{IC}_{50}$ (concentração de inibidor que produz $50 \%$ de inibição da actividade da enzima) da ordem das dezenas de microM. Esta actividade inibitória in vitro foi de seguida confirmada utilizando um sistema celular de levedura que expressa a caspase-3 humana (ensaios realizados no REQUIMTE/ CEQUP, Serviço de Microbiologia da Faculdade de Farmácia do Porto). Os resultados mostraram que as vinil sulfonas protegidas com Fmoc, e contendo apenas Asp na cadeia de reconhecimento (Figura 7, estrutura A: $G P=F m o c, R^{1}=$ metilo ou fenilo), inibem uma via dependente de caspase-3. Adicionalmente, os valores de $\mathrm{IC}_{50}$ obtidos são da mesma ordem de grandeza do inibidor tetrapeptídico de caspase-3, Ac-Asp-Glu-Val-Asp-clorometilcetona [23].

\section{EXISTE UM FUTURO PARA OS INIBIDO- Res de Caspases?}

Até à data, devido a problemas de selectividade, a maioria dos inibidores de caspases não chegaram a entrar em estudos pré-clínicos com modelos animais de patologias humanas. Apenas 4 inibidores, VX-740 (Pralnacasan), VX-765, IDN-6556 e LB84451, chegaram a entrar em ensaios clínicos, tendo dois deles, o VX-740 e o IDN-6556, sido descontinuados na fase 2 dos ensaios clínicos (Figura 8) [11].

Apesar da dificuldade observada na obtenção de um fármaco selectivo para as caspases, o conhecimento científico actual torna evidente que as caspases são um importante alvo terapêutico para a inibição da apoptose. No entanto, a inibição da apoptose terá que ser suficientemente selectiva para evitar o bloqueio da apoptose normal, necessária nos organismos multicelulares. Portanto, existe ainda um largo campo de investigação em aberto para o desenvolvimento de inibidores de caspases e para o estudo do seu potencial para o tratamento de doenças neurodegenerativas.<smiles>[R]OS(=O)C=CC(CC(=O)O)Nc1ccccc1</smiles>

A<smiles>[R]C(NCCCC)C(=O)NC(C=CS(=O)[O-])CC(=O)O</smiles>

B

Figura 7 - Vinil sulfonas sintetizadas<smiles>CCOC1CC(=O)OC1NC(=O)[C@@H]1CCCN2C(=O)CC[C@H](NC(=O)c3nccc4ccccc34)C(=O)N12</smiles>

Pralnacasan<smiles>CCOC1CC(=O)OC1NC(=O)[C@@H]1CCCN1C(=O)C(NC(=O)c1ccc(N)c(Cl)c1)C(C)(C)C</smiles>

VX-765

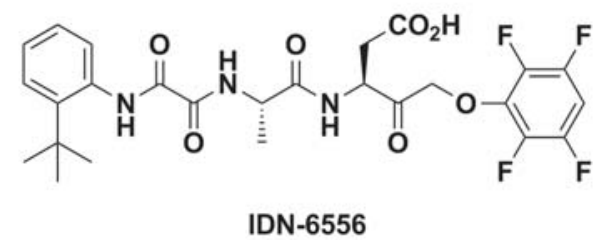

Figura 8 - Inibidores de caspases que entraram em ensaios clínicos

\section{Agradecimentos}

A autora gostaria de exprimir o seu agradecimento ao Prof. Rui Moreira por todas as discussões científicas extremamente úteis e ao Dr. Daniel Santos pelo apoio na preparação da figura 3 .

\section{REFERÊNCIAS}

[1] M.O. Hengartner, Nature 407 (2000) 770-776.

[2] T.G. Cotter, Nature Reviews Cancer 9 (2009) 501-507.

[3] R.E. Castro, M.M.M. Santos, P.M.C. Glória, C.J.A. Ribeiro, D.M.S. Ferreira, J.M. Xavier, R. Moreira, C.M.P. Rodrigues, Current Pharmaceutical Design 16 (2010) 2851-2864.

[4] J.F.R. Kerr, A.H. Wyllie, A.R. Currie, British Journal of Cancer 26 (1972) 239-257.

[5] W.C. Earnshaw, L.M. Martins, S.H. Kaufmann, Annual Review of Biochemistry 68 (1999) 383-424.

[6] J.-B. Denault, G.S. Salvesen, Chemical Reviews 102 (2002) 4489-4500.

[7] P. Fuentes-Prior, G.S. Salvesen, Biochemical Journal384(2004)201-232.

[8] C.M. Troy, G.S. Salvesen, Journal of Neuroscience Research 69 (2002) 145-150.

[9] R.M. Friedlander, The New England Journal of Medicine 348 (2003) 13651375.
[10] S.D. Linton, Current Topics in Medicinal Chemistry 5 (2005) 1697-1717.

[11] H. Eda, 'Therapeutic potential for caspase inhibitors present and future', in Design of caspase inhibitors as potential clinical agents, USA: CRC Press (2009) 251-287.

[12] T. O'Brien, D. Lee, Mini-Reviews in Medicinal Chemistry 4 (2004) 153165.

[13] J.C. Powers, J.L. Asgian, Ö.D. Ekici, K.E. James, Chemical Reviews 102 (2002) 4639-4750.

[14] R. Leung-Toung, Y. Zhao, W. Li, T.F. Tam, K. Karimian, M. Spino, Current Medicinal Chemistry 13 (2006) 547581.

[15] a) S.D. Linton, T. Aja, P.R. Allegrini, T.L. Deckwerth, J.L. Diaz, B. Hengere, J. Herrman, K.G. Jahangiri, J. Kallen, D.S. Karanewsky, S.P. Meduna, K. Nalley, E.D. Robinson, S. Roggo, G. Rovelli, A. Sauter, R.O. Sayers, A. Schmitz, R. Smidt, R.J. Ternansky, K.J. Tomaselli, B.R. Ullman, C. Wiessner, J.C. Wu, Bioorganic \& Medicinal Chemistry Letters 14 (2004) 2685-2691. b) H. Ueno, M. Kawai, H. Shimokawa, M. Hirota, M. Ohmi, R. Sudo, A. Ohta, Y. Arano, K. Hattori, T. Ohmi, N. Kato, M. Kojima, Y. Ueno, M. Yamamoto, Y. Moriguchi, H. Eda, K. Masubuchi, Bioorganic \& Medicinal Chemistry Letters 19 (2009) 199-202. 
[16] W. Yang, J. Guastella, J.-C. Huang, Y. Wang, L. Zhang, D. Xue, M. Tran, R. Woodward, S. Kasibhatla, B. Tseng, J. Drewe, S.X. Cai, British Journal of Pharmacology 140 (2003) 402-412.

[17] J. Rotonda, D.W. Nicholson, K.M. Fazil, M. Gallant, Y. Gareau, M. Labelle, E.P. Peterson, D.M. Rasper, R. Ruel, J.P. Vaillancourt, N.A. Thornberry, J.W. Becker, Nature Structural Biology 3 (1996) 619-625.

[18] a) D. Lee, S.A. Long, J.H. Murray, J.L. Adams, M.E. Nuttall, D.P. Nadeau, K. Kikly, J.D. Winkler, C.M. Sung, M.D. Ryan, M.A. Levy, P.M. Keller, W.E.Jr. DeWolf, Journal of Medicinal Chemistry 44 (2001) 2015-2026. b) W.
Chu, J. Zhang, C. Zeng, J. Rothfuss, Z. Tu, Y. Chu, D.E. Reichert, M.J. Welch, R.H. Mach, Journal of Medicinal Chemistry 48 (2005) 7637-7647.

[19] K.E. James, J.L. Asgian, Z.Z. Li, O.D. Ekici, J.R. Rubin, J. Mikolajczyk, G.S. Salvesen, J.C. Powers Journal of MedicinalChemistry47(2004)1553-1574.

[20] M.M.M. Santos, R. Moreira, MiniReviews in Medicinal Chemistry 7 (2007) 1040-1050.

[21] Ö.D. Ekici, Z.Z. Li, A.J. Campbell, K.E. James, J.L. Asgian, J. Mikolajczyk, G.S. Salvesen, R. Ganesan, S. Jelakovic, M.G. Grütter, J.C. Powers, Journal of Medicinal Chemistry 49 (2006) 5728-5749.
[22] a) W. Chu, J. Rothfuss, A. d'Avignon, C. Zeng, D. Zhou, R.S. Hotchkiss, R.H. Mach, Journal of Medicinal Chemistry 50 (2007) 3751-3755. b) W. Chu, J. Rothfuss, Y. Chu, D. Zhou, R.H. Mach, Journal of Medicinal Chemistry 52 (2009) 2188-2191.

[23] a) A.S. Newton, P.M.C. Glória, L.M. Gonçalves, D.J.V.A. dos Santos, R. Moreira, R.C. Guedes, M.M.M. Santos, European Journal of Medicinal Chemistry 45 (2010) 3858-3863. b) P.M.C. Glória, I. Coutinho, L.M. Gonçalves, C. Baptista, J. Soares, A.S. Newton, R. Moreira, L. Saraiva, M. M. M. Santos, European Journal of Medicinal Chemistry 46 (2011) 2141$-2146$.

\section{Actualidades Científicas}

\section{. .}

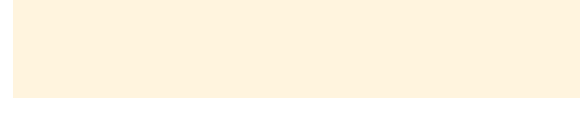

Um novo estudo demonstra que um complexo organometálico assimétrico, constituído por um átomo de érbio ladeado por dois hidrocarbonetos cíclicos diferentes, exibe propriedades magnéticas únicas.

Este complexo pode tornar-se num protótipo para o posterior desenvolvimento de magnetos unimoleculares (single-molecule magnets - SMM's), cujas aplicações se estendem desde o armazenamento de informação de alta densidade até à computação quântica.

Os magnetos convencionais dependem do comportamento conjunto dos spins de electrões desemparelhados provenientes de milhões de átomos metálicos individuais, suportados numa estrutura organizada. Por outro lado, os SMM's exibem individualmente por si só um comportamento do tipo magnético. Um aparelho magnético construído a partir destes compostos,

\section{Magnetos Unimoleculares}

em que cada molécula SMM armazena um bit de informação, pode acomodar uma quantidade de dados milhares de vezes superior à dos mecanismos de armazenamento actuais.

A maioria dos SMM's é baseada em aglomerações com múltiplos núcleos ião-metal, como $\mathrm{Mn}_{12} \mathrm{O}_{12}$. No entanto, apenas são conhecidos até ao momento cerca de 10 exemplos de SMM's de ião-metal único. Assim, uma equipa de investigação liderada por Song Gao e Bing-Wu Wang, da Universidade de Pequim, na China, sintetizou e estudou as propriedades magnéticas de um novo tipo de SMM: um complexo de érbio ciclooctatrieno pentametiIciclopentadieno (J. Am. Chem. Soc., DOI: 10.1021/ja200198v).

Este complexo de érbio é notável, já que se constitui como o primeiro exemplo de um magneto ião-metal organometálico de núcleo único; os casos reportados até ao momento continham ligandos baseados em oxigénio ou nitrogénio. Por outro lado, o anel $\mathrm{C}_{8}$ está mais próximo do átomo de érbio do que o anel $C_{5}$, e os anéis não estão perfeitamente paralelos entre si, ao contrário do que é comum nos compostos sandwich típicos.

Gao e colaboradores verificaram que esta orientação possibilita que a configuração electrónica do érbio gere propriedades magnéticas ainda não observadas em SMM's anteriores: apresenta dois processos de relaxação magnética térmica, em vez de um, e o magnetismo mantém-se numa gama de temperatura elevada.

(adaptado do artigo de 28/03/2011 de Stephen K. Ritter: Single-Molecule Magnets Evolve, Chemical \& Engineering News - http://pubs.acs.org/cen/news/89/ i13/8913notw2.html)

Paulo Brito (paulo@ipb.pt) Instituto Politécnico de Bragança

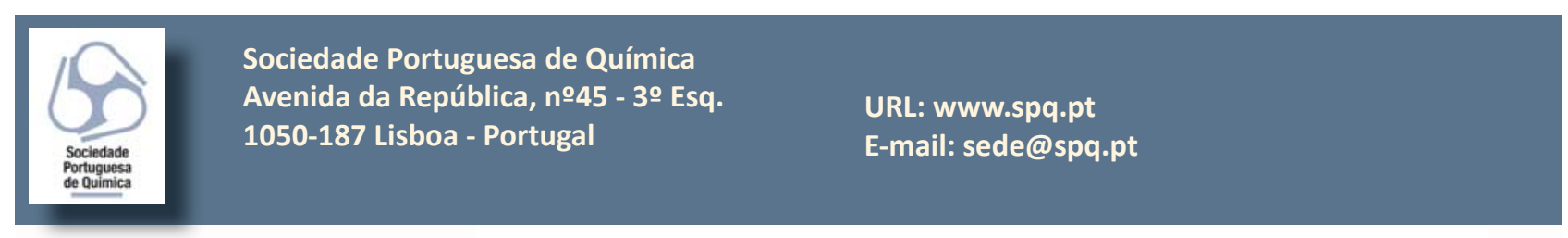




\section{Top Chemistry}

Global

\section{Visibility}
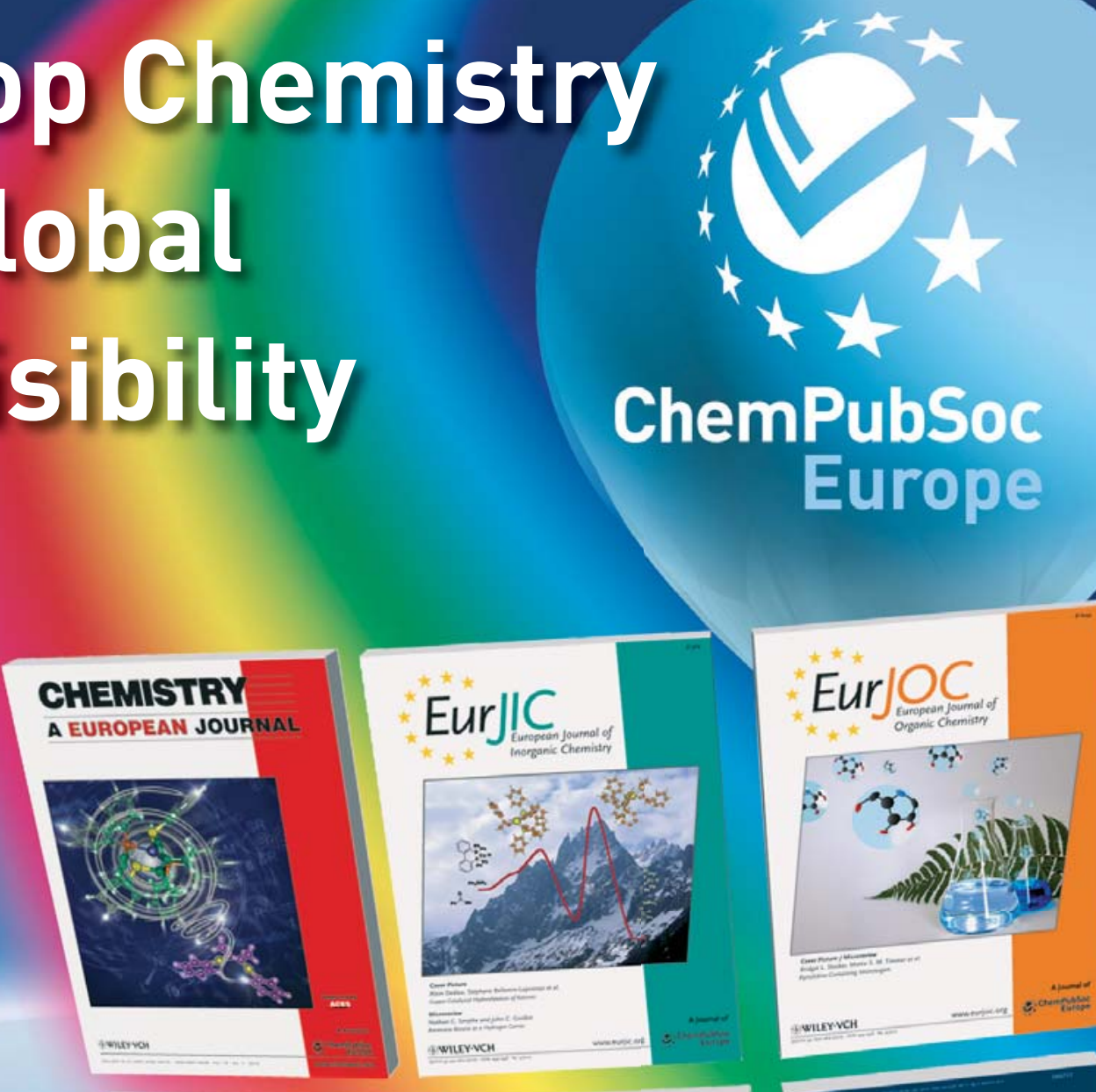

CHEMCATCHEM
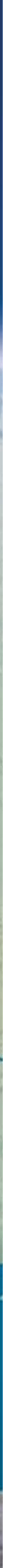

(2)WILEY-VCH

www.chemptibsoc.eu 